\title{
Supplemental vitamin D in quiescent Crohn's disease - effects on quality of life, fatigue and muscle strength: results from a double blind placebo controlled study
}

\author{
T. Raftery ${ }^{1}$, C. S. Lee ${ }^{2}$, G. Cox ${ }^{3}$, D. McNamara ${ }^{2}$, M. Healy ${ }^{3}$ and M. O'Sullivan ${ }^{1}$ \\ ${ }^{1}$ Dept of Medicine, Trinity Centre for Health Science, St. James's Hospital, Dublin 8, ${ }^{2}$ Dept. of Medicine, Tallaght Hospital, \\ Dublin 24, Ireland and ${ }^{3}$ Dept. of Biochemistry, St. James's Hospital, Dublin 8, Ireland
}

Reduced muscle strength, fatigue and poor quality of life (QoL) are frequently reported in Crohn's disease (CD) even in remission ${ }^{(1)}$. There is growing evidence that vitamin D (vitD) may alter skeletal muscle function and strength ${ }^{(2)}$. Our aim was to investigate the effects of vitD supplementation on muscle function and, correspondingly, on fatigue and QoL in patients with stable CD. In a double-blind placebo controlled study, 27 subjects with $\mathrm{CD}$ in remission were randomized to $2000 \mathrm{IU}$ vitD3/d or placebo for 3 mo. The primary outcome measures were changes in hand-grip strength (HGS) (Jamar Digital Hand Dynamometer), fatigue (Multi-fatigue inventory, $\mathrm{MFI}$ ) and QoL (Inflammatory Bowel Disease questionnaire, IBDQ). At 0 and 3mo, disease activity (CD activity index, CDAI), FBC, CRP $(\mathrm{mg} / \mathrm{l})$ and serum $25(\mathrm{OH}) \mathrm{D}(\mathrm{nmol} / \mathrm{l})$ were also measured. Ethical approval and informed written consent were obtained and the research was performed in accordance to the Declaration of Helsink.

At enrolment mean (sem) CRP [2.39 (0.53)] and CDAI [92.35 (13.23)] were consistent with disease remission and Hb and haemocrit were within normal ranges. At 3mo, serum 25(OH)D increased significantly in the treated group [69.2 (7.0) to 91.6 (25.3) nmol/l, $p=0.002$ ] and decreased in the controls [51.8 (20.7) to $40.4(14.9) \mathrm{nmol} / \mathrm{l}, p=0.021]$. Baseline HGS was matched in the VitD-treated and placebo groups, with no difference in either dominant [treated: $80.2(7.8)$; placebo: $66.7(7.6), p=0.2$ ] or non-dominant HGS between the groups [treated: $78.6(9.9)$, placebo: $64.8(7.2), p=0.26)$. Post-intervention, both dominant and non-dominant HGS were significantly higher in the VitD-treated group [dominant HGS; 94.1 (8.6)v. 63.6 (8.3), $p=0.0191$, non-dominant HGS; 90.7 \pm 9.3 v.60.5 (7.6), $p=0.0209$ ].

At $3 \mathrm{mo}$, patients who achieved $25(\mathrm{OH}) \mathrm{D}$ levels $>=75 \mathrm{nmol} / \mathrm{l}$ had significantly higher QoL [mean (sem)] compared to patients below this cut-off [IBDQ score 187.3 (2.3) v. $163.2(2.1), p=<0.0001$ ]. Furthermore, bowel and social domains of the IBDQ showed significant improvement in those achieving $>=75 \mathrm{nmol} / \mathrm{l} 25(0 \mathrm{H}) \mathrm{D}$ compared to those who did not [Bowel $60.5(1.9)$ v. $51.5(2.7), p=0.015 ;$ Social 59.4 (6.3) v. 44.5 (4), $p=0.05$ ]. In line with this, significantly less fatigue [mean (sem)] was experienced in those with $25(\mathrm{OH}) \mathrm{D}$ levels $>=75 \mathrm{nmol} / \mathrm{l} \mathrm{v}$. those below this cut-off, as assessed by both question 2 of the IBDQ [5.0 (0.4) v. $3.6(0.37)$ respectively, $p=0.032$ ] and the MFI [20.4 (1.3) v $26.5(3.1), p=0.08]$. This finding was consistent in each of the MFI sub-domains, namely physical and general fatigue, activities of daily living and mental fatigue.

In conclusion, oral daily supplementation with $2000 \mathrm{IU}$ vitd3/d for $3 \mathrm{mo}$ significantly increased dominant and non-dominant HGS compared to placebo. Achieving serum 25(OH)D levels of $75 \mathrm{nmol} / \mathrm{l}$ or greater was associated with increased QOL and reduced fatigue scores. These findings, to our knowledge, are the first to suggest potential benefits of vitD on muscle strength with corresponding benefits for fatigue and QOL in $\mathrm{CD}$, and merit investigation in larger controlled studies.

1. Valentini L, Schaper L, Buning C et al. (2008) Nutrition 24(7-8), 694-702.

2. van Langenberg DR. (2013) Dig Dis Sci 58(2), 293-5. 